\title{
Sarcopenia prevalence: reply to comment by Perez-Zepeda et al
}

\author{
R. Patil • K. Uusi-Rasi • M. Pasanen • P. Kannus • \\ S. Karinkanta $\cdot$ H. Sievänen
}

Received: 4 July 2012 / Accepted: 4 July 2012 /Published online: 1 August 2012

(C) International Osteoporosis Foundation and National Osteoporosis Foundation 2012

\section{Dear Editor,}

As we discussed in our paper [1], our study population consisted of 70- to 80-year-old home-dwelling women who voluntarily participated in the DEX randomized controlled trial [2], and it is likely that the prevalence of sarcopenia in the unselected Finnish population of elderly women would have been higher than that reported by us.

We estimated muscle mass with dual-energy X-ray absorptiometry, which is the preferred method for research and clinical use [3]. In the study by ArangoLopera and colleagues, muscle mass was determined by calf circumference [4]. Diagnostic criteria (including

This reply refers to the comment available at DOI: $10.1007 / \mathrm{s} 00198$ 012-2091-x.

R. Patil $(\bowtie) \cdot$ K. Uusi-Rasi $\cdot$ M. Pasanen $\cdot$ P. Kannus •

S. Karinkanta $\cdot H$. Sievänen

The UKK Institute for Health Promotion Research,

Tampere, Finland

e-mail: radhika.patil@uta.fi

K. Uusi-Rasi

Research Department, Tampere University Hospital,

Tampere, Finland

P. Kannus

Medical School, University of Tampere,

Tampere, Finland

P. Kannus

Division of Orthopaedics and Traumatology,

Department of Trauma, Musculoskeletal Surgery and Rehabilitation,

Tampere University Hospital,

Tampere, Finland those used in the European Working Group on Sarcopenia in Older People algorithm) need to be standardized and consistently applied before they can be deemed worthy of comparison. Unless this is done, diagnosis and prevalence rates of sarcopenia are difficult to compare and do not hold credibility.

We also explored the rationale behind measuring muscle mass to predict the onset of disability in older adults. The result was that muscle mass and derived indices of sarcopenia were not related to measures of physical function. It seemed that an appropriate and standardized functional ability test battery might be better suited to detect changes in physical function and, consequently, reveal the onset of disability.

\section{References}

1. Patil R, Uusi-Rasi K, Pasanen M, Kannus P, Karinkanta S, Sievänen H (2012) Sarcopenia and osteopenia among 70-80-year-old homedwelling Finnish women: prevalence and association with functional performance. Osteoporos Int. doi:10.1007/s00198-012-2046-2

2. Uusi-Rasi K, Kannus P, Karinkanta S, Pasanen M, Patil R, Lamberg-Allardt C, Sievänen H (2012) Study protocol for prevention of falls: a randomized controlled trial of effects of vitamin D and exercise on falls prevention. BMC Geriatr 12:12. doi:10.1186/14712318-12-12

3. Cruz-Jentoft A, Baeyens J, Bauer J, Boirie Y, Cederholm T, Landi F, Martin F, Michel J, Rolland Y, Schneider S, Topinkova E, Vandewoude M, Zamboni M (2010) Sarcopenia: European consensus on definition and diagnosis. Report of the European Working Group on Sarcopenia in Older People. Age Ageing 39:412-423

4. Arango-Lopera VE, Arroyo P, Gutiérrez-Robledo LM, PérezZepeda MU (2012) Prevalence of sarcopenia in Mexico City. European Geriatric Medicine 3:157-160 